\title{
Senescence of Rice Leaves XXX. Levels of Endogenous Polyamines and Dark-Induced Senescence of Rice Leaves
}

\author{
Chien Teh Chen and Ching Huei Kao
}

\author{
Department of Agronomy, National Taiwan University, Taipei, Taiwan, Republic of China
}

\begin{abstract}
The role of endogenous polyamines in the control of dark-induced senescence of detached rice leaves was investigated by quantitating levels of various polyamines by HPLC. Putrescine, spermidine and spermine were all present throughout senescence. Neither cadaverine nor 1,3diaminopropane was detected. During dark-induced senescence, there was a marked decrease in levels of putrescine and an increase in those of spermidine and spermine. The rate of production of ethylene increased markedly upon excision of leaves. $a$-Difluoromethylarginine (DFMA) and $a$-difluoromethylornithine (DFMO) caused a reduction in levels of putrescine, yet had no effect on levels of spermidine and spermine. Neither DFMA nor DFMO had any effect on senescence or on the production of ethylene. Treatment with dicyclohexylamine (DCH) and methylglyoxal bis-(guanylhydrazone) (MGBG) reduced levels of spermine and increased those of putrescine in detached leaves. After treatment with DCH or MGBG, both senescence and the production of ethylene were significantly promoted. The current results suggest that endogenous polyamines may not play a significant role in the control of dark-induced senescence of rice leaves. This conclusion is supported by the further observations that (a) benzyladenine, which is known to retard senescence, decreased levels of putrescine but had no effect on those of spermidine and spermine; and (b) ABA, which promoted senescence, increased levels of putrescine and had no effect on those of spermidine and spermine.
\end{abstract}

Key words: S-adenosylmethionine decarboxylase - Arginine decarboxylase - Ethylene - Ornithine decarboxylase - Polyamines - Rice leaf senescence.

In recent years, increasing attention has been paid to the possible role of polyamines in growth and differentiation (Evans and Malmberg 1989). Polyamines are effective in retarding senescence of excised leaves and protoplasts (Altman 1982, Cheng and Kao 1983, Galston et al. 1978, Kaur-Sawhney and Galston 1979). Since decreases in levels of polyamines have been observed in aging and senescing leaves, it is reasonable to suppose that they play a role in the regulation of leaf senescence (Kaur-Sawhney et ai. 1982, Srivastava 1981). However, a decrease in levels of polyamines is not always correlated with the onset of leaf senescence (Birecka et al. 1984). One way to approach

Abbreviations: ADC, arginine decarboxylase; BA, benzyladenine; Cad, cadaverine; Dap, 1,3-diaminopropane; DCH, dicyclohexylamine; DFMA, $a$-difluoromethylarginine; DFMO, $a$ difluoromethylornithine; MGBG, methylglyoxal bis-(guanylhydrazone); ODC, ornithine decarboxylase; Put, putrescine; SAM, S-adenosylmethionine; SAMDC, S-adenosylmethionine decarboxylase; Spd, spermidine; Spm, spermine. the question of whether or not polyamines have a functional role in the control of senescence involves the use of inhibitors of polyamine synthesis. Using this approach, Birecka et al. (1990) showed that dark-induced leaf senescence of barley and oats was not correlated with the levels of endogenous polyamines. The present investigation was conducted to determine the role of endogenous polyamines in the control of senescence of detached rice leaves. The mechanism by which exogenous polyamines retard leaf senescence may be related to the possibility that they inhibit the biosynthesis of ethylene (Evans and Malmberg 1989). Both ethylene and polyamines share SAM as a common precursor (Miyazaki and Yang 1987). Thus, changes in rates of production of ethylene during dark-induced senescence of detached rice were also investigated.

\section{Materials and Methods}

Plant material-Rice (Oryza sativa L. cv. Taichung Native 1) seedlings were grown as previously described 
(Chen et al. 1990). The apical $3 \mathrm{~cm}$ of the third leaves of 10-day-old seedlings were used for experiments. Ten segments of rice leaves were floated on $10 \mathrm{ml}$ of distilled water or of a test solution in a Petri dish. All samples were incubated at $27^{\circ} \mathrm{C}$ in the dark.

Chl determination-Chl was determined by the method of Wintermans and De Mots (1965) after extraction in $96 \%$ ethanol. Chl content is expressed as mg per $\mathrm{g}$ fresh weight.

Protein determination-Leaf segments were homogenized in $25 \mathrm{~mm}$ sodium phosphate buffer (pH 7.5). The extracts were centrifuged at $17,000 \times g$ or $20 \mathrm{~min}$, and the supernatants were used for determination of protein by the method of Lowry et al. (1951).

Polyamine determinations-Leaf segments weighing about $125 \mathrm{mg}$ were homogenized in $5 \mathrm{ml}$ of cold $5 \%$ perchloric acid, and the homogenates were kept at $4^{\circ} \mathrm{C}$ for $24 \mathrm{~h}$. Each homogenate was centrifuged for $20 \mathrm{~min}$ at $17,000 \times g$ and the clear supernatant was used for benzoylation according to Flores and Galston (1982). In brief, $1 \mathrm{ml}$ of $\mathrm{NaOH}(2 \mathrm{~N})$ was mixed with the $1 \mathrm{ml}$ of the supernatant. After addition of $15 \mu \mathrm{l}$ of benzoyl chloride, vortexing for $10 \mathrm{~s}$, and incubation for $20 \mathrm{~min}$ at room temperature (about $25^{\circ} \mathrm{C}$ ), $2.5 \mathrm{ml}$ of saturated $\mathrm{NaCl}$ were added. Benzoyl-polyamines were extracted in $2.5 \mathrm{ml}$ of diethylether. After centrifugation at $3,000 \times g$ for $15 \mathrm{~min}, 1.5 \mathrm{ml}$ of the ether phase were collected, evaporated to dryness in a vacuum concentrator and redissolved in $100 \mu \mathrm{l}$ of methanol. Polyamine standards were benzoylated in a similar way. Ten $\mu$ laliquots of each redissolved sample were injected into a Waters M-6 UK Universal Liquid Chromatograph. They were eluted through a $4 \times 250 \mathrm{~mm}, 5 \mu \mathrm{m}$ particle size $C 18$ reverse-phase column at a flow rate of $1 \mathrm{ml}$ $\min ^{-1}$ using a water (solvent $A$ )/methanol (solvent B) stepped gradient program (50 to $65 \% \mathrm{~B}$ in $7 \mathrm{~min} / 65$ to $80 \% \mathrm{~B}$ in $10.5 \mathrm{~min} / 80$ to $100 \% \mathrm{~B}$ in $2 \mathrm{~min}$ ) followed by a column cleaning/regeneration cycle. Detection was accomplished by a Waters M481 absorbance detector at $254 \mathrm{~nm}$. Results were recorded on a Waters M740 data module and plotted as $\mathrm{nmol}$ per $\mathrm{g}$ fresh weight.

Ethylene determination-Leaf segments were placed vertically in test tubes which were closed with rubber stoppers. After $1 \mathrm{~h}$ of incubation at $27^{\circ} \mathrm{C}$ in the dark, a $1-\mathrm{ml}$ sample of gas was withdrawn from the headspace of each test tube. Ethylene was assayed using a gas chromatograph equipped with an alumina column and a flame ionization detector.

\section{Results}

The senescence of detached rice leaves was followed by measuring the decrease in levels of $\mathrm{Chl}$ and the protein. Figure 1 shows that a decrease in levels of $\mathrm{Chl}$ and protein was evident at 2 and 3 days, respectively, after leaf detach-

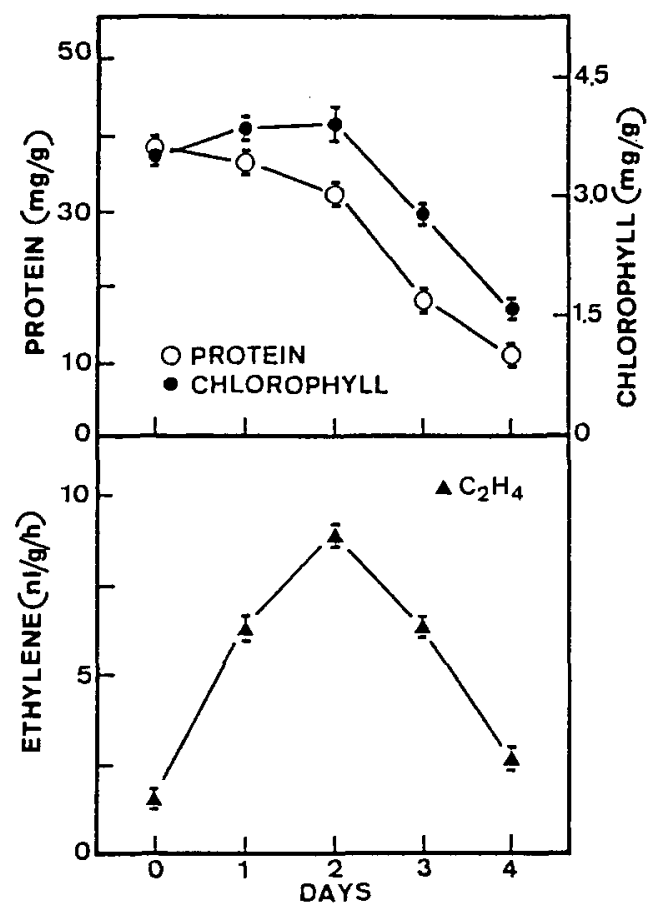

Fig. 1 Changes in levels of chlorophyll and protein and in rates of production of ethylene in detached rice leaves incubated in darkness. Means $\pm \mathrm{SE}, 4$ replicates.

ment. This indicates that proteolysis precedes $\mathrm{Chl}$ degradation during senescence of detached rice leaves in the dark, in good agreement with the results of Shibaoka and Thimann (1970) and our earlier work (Kao 1978).

In freshly excised rice leaf segments, ethylene production was low (Fig. 1). After incubation, the rate of ethylene production increased, reaching a maximum in 2 days, and subsequently declined (Fig. 1). The rise of ethylene production occurred prior to senescence.

A typical chromatogram of benzoyl derivatives obtained from freshly excised leaves, with authentic standards included for comparison is shown in Figure 2. The major peaks corresponded to Put, Spd and Spm. Peaks in leaf extracts corresponding to authentic Cad and Dap were not observed. The level of Put in detached rice leaves decreased progressively with increasing duration of incubation in darkness (Fig. 3). However, the level of Spd increased immediately after leaf detachment, reached a maximum level at days 1 and 2, and then decreased to the basal level (Fig. 3). The level of Spm also increased immediately after excision of leaves, reached maximum level at day 2 , and remained unchanged thereafter (Fig. 3).

The time courses of changes in levels of $\mathrm{Chl}$ and protein in detached leaves floating on water and on a solution of BA, a synthetic cytokinin, in darkness, are shown in Figure 4. It is clear that BA retards senescence of detached 


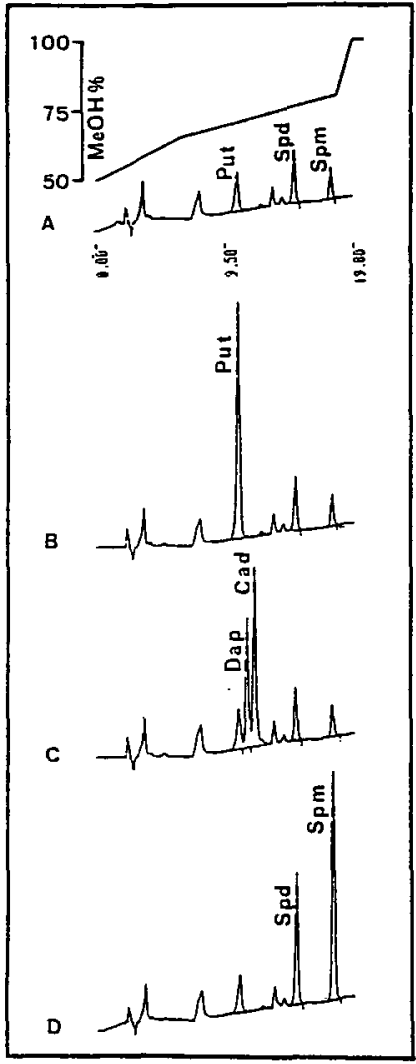

Fig. 2 Separation of benzoylated diamines and polyamines by HPLC. A, Extract of leaves of 10-day-old seedlings; B, leaf extract with authentic Put; C, leaf extract with authentic Cad and Dap; D, leaf extract with authentic Spd and Spm. All authentic standards were present at $1.5 \mathrm{nmol}$. Numbers below trace $\mathrm{A}$ are retention times.

leaves. However, BA had no effect on levels of Spd and Spm, but it did decrease the level of Put over the first two days (Fig. 5). Figure 6 shows the effects of ABA on the senescence of detached leaves. ABA significantly promoted senescence. The effect of ABA on levels of polyamines is shown in Figure 7. ABA significantly increased the level of Put. However, no significant difference in levels of Spm in detached leaves was observed between those treated with ABA and controls in water. The decrease in level of Spd only occurred four days after the start of treatment with ABA. The observed effects of BA and ABA on levels of Put in detached rice leaves are in sharp contrast to those reported by other investigators (Mukhopadhyay et al. 1983, Suresh and Adiga 1978, Suresh et al. 1978), who reported that cytokinin and ABA increased and decreased levels of Put, respectively.

To characterize further the role of endogenous polyamines in dark-induced senescence, inhibitors of the biosynthesis of polyamines, such as DFMO, DFMA, MGBG and

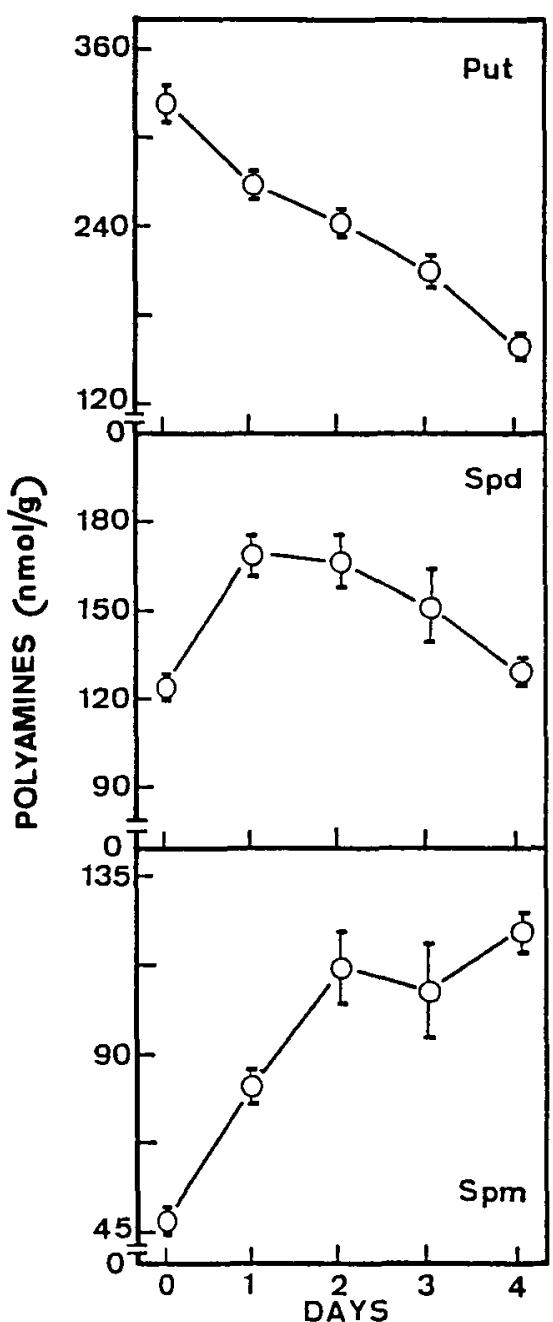

Fig. 3 Changes in levels of polyamines in detached rice leaves incubated in darkness. Mean \pm SE, 3 replicates.

$\mathrm{DCH}$, were used to change level of intracellular polyamines in detached rice leaves. Both DFMO, an irreversible inhibitor of ODC, and DFMA, an irreversible inhibitor of ADC, significantly reduced levels of Put, yet neither had any effect on levels of Spd and Spm (Table 1). The biosynthesis of Put in the aleurone layers of barley seeds was not inhibited by DFMO or DFMA (Lin 1984). However, our results are consistent with those reported by Roberts et al. (1984). DFMA was more effective in reducing the level of Put than was DFMO, indicating that the biosynthesis of Put in rice leaves occurs mainly via the reaction catalyzed by ADC. However, neither DFMA nor DFMO had any effect on the levels of $\mathrm{Chl}$ and protein or on the rate of production of ethylene (Tables 1 and 2). DCH and MGBG reduced levels of Spm in detached rice leaves (Table 1). There was also an increase in levels of Put in the DCH- and MGBG-treated detached rice leaves. Neither DCH nor MGBG affected 


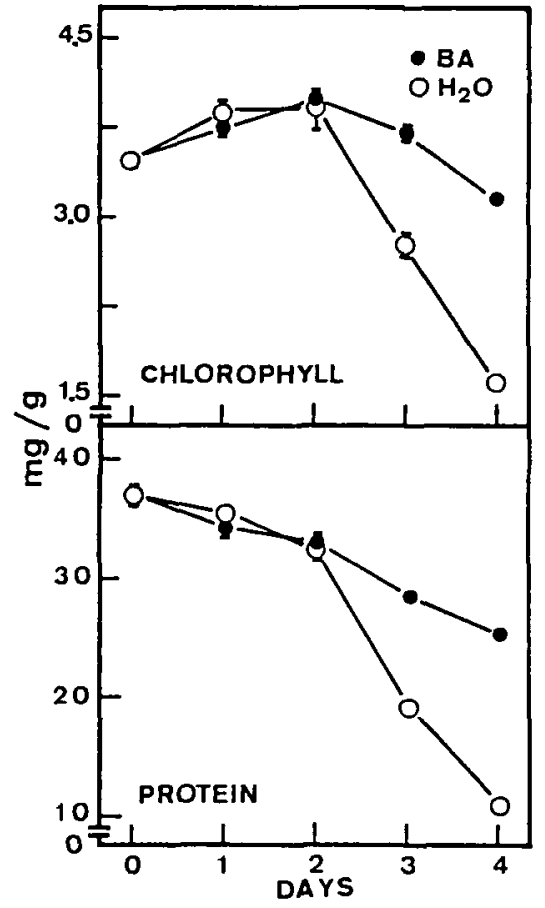

Fig. 4 Effects of BA on levels of $\mathrm{Chl}$ and protein in detached rice leaves incubated in darkness. Means $\pm S E, 4$ replicates.

levels of Spd (Table 1) but they both caused significant decreases in levels of Chl and protein and increased rates of production of ethylene (Tables 1 and 2 ).

\section{Discussion}

The present investigation showed that Put, Spd and Spm were present in detached rice leaves throughout senescence. However, neither Cad nor Dap was detected in detached rice leaves during dark-induced senescence, indicating that Cad and Dap may not be involved in the control of dark-induced senescence of detached rice leaves.

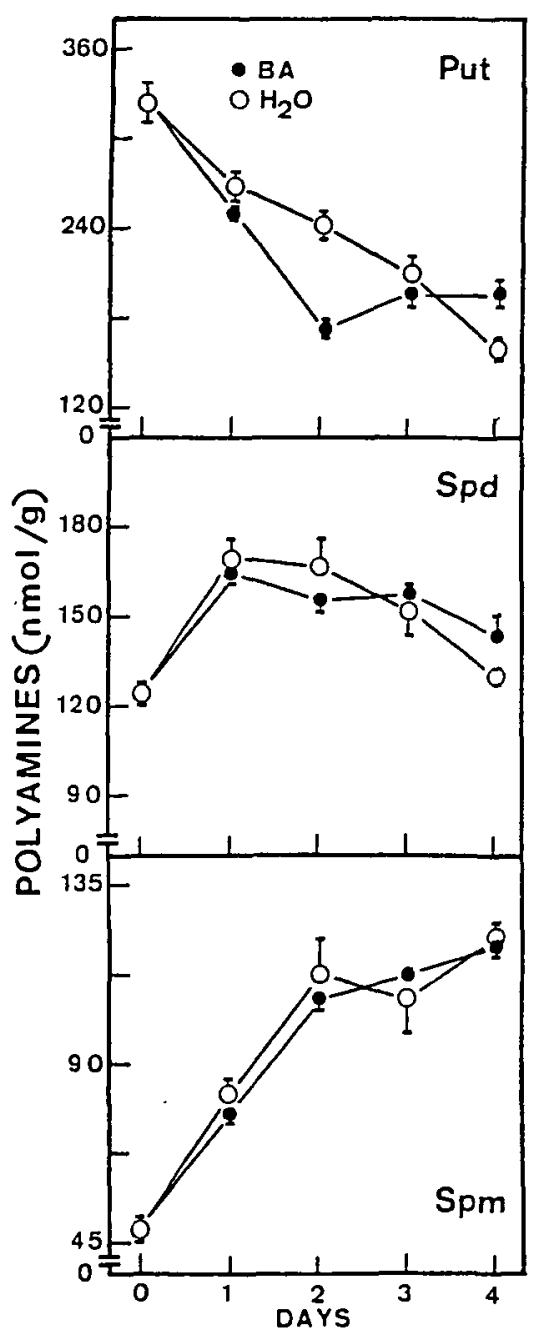

Fig. 5 Effects of BA on levels of polyamine in detached rice leaves incubated in darkness. Means $\pm \mathrm{SE}, 3$ replicates.

The absence of Cad and Dap has also been reported in pea ovaries during senescence (Carbonell and Navarro 1989).

Table 1 Effects of DFMO, DFMA, DCH and MGBG on levels of polyamines and the rate of production of ethylene in detached rice leaves incubated in darkness

\begin{tabular}{|c|c|c|c|c|}
\hline \multirow{2}{*}{ Treatment } & \multirow{2}{*}{$\begin{array}{c}\text { Ethylene } \\
\left(\text { nl } g^{-1} h^{-1}\right)\end{array}$} & Put & Spd & Spm \\
\hline & & \multicolumn{3}{|c|}{$\left(\mathrm{nmol} \mathrm{g}^{-1}\right)$} \\
\hline Control & $6.4 \pm 0.2$ & $250 \pm 3$ & $153 \pm 2$ & $50 \pm 2$ \\
\hline DFMO, $0.5 \mathrm{mM}$ & $6.0 \pm 0.3$ & $240 \pm 4$ & $148 \pm 2$ & $59 \pm 5$ \\
\hline DFMA, $0.5 \mathrm{mM}$ & $6.2 \pm 0.4$ & $126 \pm 10$ & $155 \pm 9$ & $53 \pm 3$ \\
\hline Control & $6.4 \pm 0.2$ & $176 \pm 9$ & $130 \pm 4$ & $98 \pm 1$ \\
\hline $\mathrm{DCH}, 2.5 \mathrm{mM}$ & $8.0 \pm 0.2$ & $234 \pm 6$ & $137 \pm 2$ & $86 \pm 3$ \\
\hline MGBG, $5.0 \mathrm{~mm}$ & $8.8 \pm 0.2$ & $240 \pm 1$ & $123 \pm 1$ & $64 \pm 2$ \\
\hline
\end{tabular}

Rates of production of ethylene and levels of polyamines were determined $24 \mathrm{~h}$ after the start of incubation. 


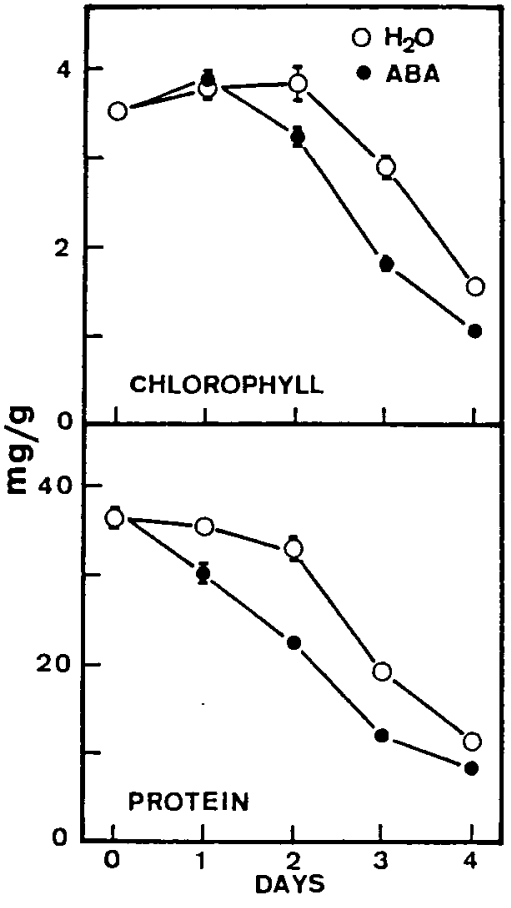

Fig. 6 Effects of $\mathrm{ABA}$ on levels of $\mathrm{Chl}$ and protein in detached rice leaves incubated in darkness. Means $\pm S E, 4$ replicates.

Exogenous applications of Spd and Spm retard senescence of detached rice leaves (Cheng and Kao 1983). If Spd and Spm play a role in the regulation of senescence of leaves, a decrease in levels of Spd and/or Spm would be expected. However, such a decrease was not observed. The increase in levels of Spd and Spm observed in the present study simply reflects the changing metabolic requirements after detachment of leaves.

Our results shows that levels of Put in detached rice leaves decrease with increasing duration of dark incubation. Moreover, the decrease in levels of Put occurs prior to loss of $\mathrm{Chl}$ and degradation of protein. Although it seems possible that Put might be involved in the control of senescence of detached rice leaves, this possibility was not confirmed by the results of the application of inhibitors of the biosynthesis of polyamines to detached rice leaves. DFMA and DFMO significantly decreased levels of Put without affecting senescence of detached rice leaves. By contrast, treatments with DCH and MGBG resulted in elevated levels of Put in detached rice leaves but also promoted senescence. The evidence presented here leads to the conclusion that a lowering of the level of Put is unlikely to be the factor responsible for the senescence of detached rice leaves in darkness. This conclusion is further supported by the observations that (a) BA, which retards senescence of detached rice leaves in the dark, decreased levels of Put; and (b) ABA, which promots senescence, increased

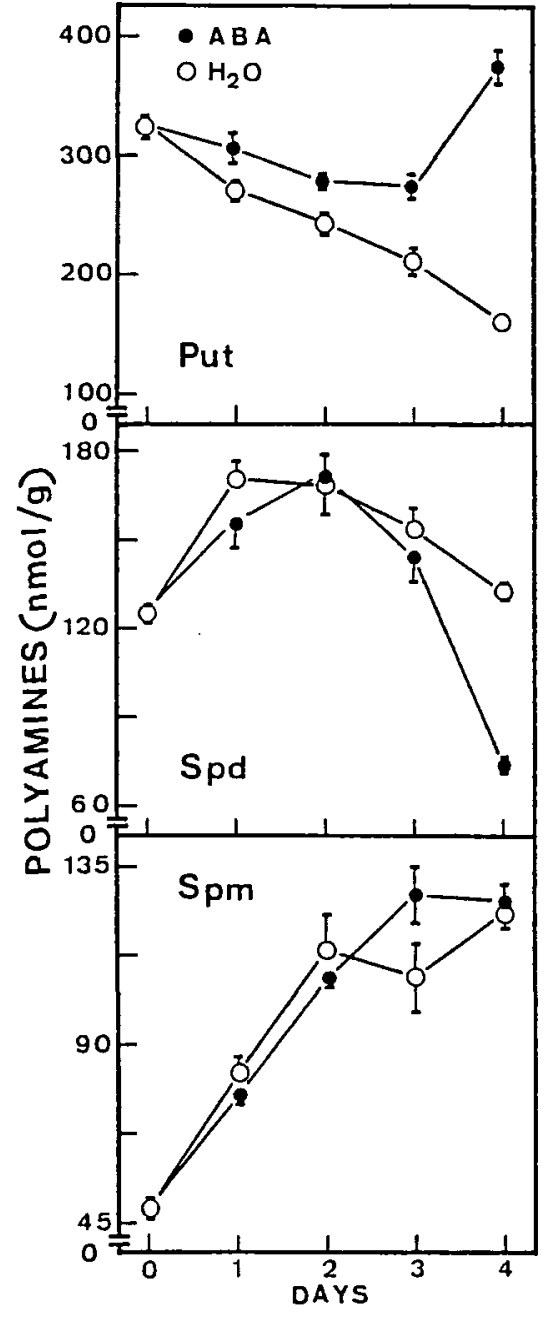

Fig. 7 Effects of ABA on levels of polyamines in detached rice leaves incubated in darkness. Means $\pm \mathrm{SE}, 3$ replicates.

Table 2 Effects of DFMO, DFMA, DCH and MGBG on levels of $\mathrm{Chl}$ and protein in detached rice leaves incubated in darkness

\begin{tabular}{lcc}
\hline Treatment & $\begin{array}{c}\text { Chl } \\
\left(\mathrm{mg} \mathrm{g}^{-1}\right)\end{array}$ & $\begin{array}{c}\text { Protein } \\
\left(\mathrm{mg} \mathrm{g}^{-1}\right)\end{array}$ \\
\hline Control & $2.6 \pm 0.1$ & $20 \pm 1$ \\
DFMO, 0.5 mM & $2.4 \pm 0.1$ & $22 \pm 1$ \\
DFMA, 0.5 mM & $2.8 \pm 0.2$ & $19 \pm 1$ \\
Control & $2.6 \pm 0.1$ & $20 \pm 1$ \\
DCH, $2.5 \mathrm{mM}$ & $0.9 \pm 0.1$ & $13 \pm 1$ \\
MGBG, $5.0 \mathrm{mM}$ & $1.5 \pm 0.1$ & $16 \pm 1$ \\
\hline
\end{tabular}

Levels of $\mathrm{Chl}$ and protein levels were determined 3 days after the start of incubation. 
the level of Put. Birecka et al. $(1984,1990)$ also reported that a decline in levels of polyamines was not correlated with the onset of senescence. The present investigation provides support for the suggestion of Birecka et al. (1984) that endogenous polyamines may have effects that differ from the possibly nonspecific effects frequently reported for exogenous polyamines.

MGBG, an inhibitor of SAMDC in bacterial and mammalian cells (Willams-Ashman and Schenone 1972), has recently been used in a number of investigations on polyamine metabolism in plants. This compound inhibits SAMDC activity and reduces levels of Spd in wild carrot and maize (Feirer et al. 1985, Hirawasa and Suzuki 1983). Although we have demonstrated that SAMDC activity from rice leaves is strongly inhibited by MGBG (data not shown), it was the level of Spm rather than that of Spd that was reduced by the treatment with MGBG. DCH is a competitive inhibitor of Spd synthase (Hibasami et al. 1980). The inhibition of Spd synthase by DCH causes a reduction in levels of both Spd and Spm in cultured cotyledons of Pinus radiata and germinating seeds of Acer (Biondi et al. 1988, Walker et al. 1985). In other systems no changes or increases in levels of Spm have been observed (Mitchell et al. 1985, Torrigiani et al. 1987). In the current study we found, however, that only levels of Spm were reduced by treatment of rice leaves with $\mathrm{DCH}$. There is one report that DCH is not an inhibitor of Spd synthase (Batchelor et al. 1986).

Since ethylene and polyamines share SAM as a common precursor (Miyazaki and Yang 1987), the changes in levels of polyamines should be inversely correlated with changes in rates of production of ethylene during senescence of detached rice leaves. However, this corrleation was not observed, and it appears that biosynthesis of polyamines and ethylene does not involve active competition for the substrate, SAM, during the course of senescence.

A dramatic increase in the rate of production of ethylene was observed prior to senescence of detached rice leaves, an observation that is in good agreement with our earlier conclusion that senescence of detached rice leaves is directly linked to the production of ethylene (Kao and Yang 1983). This conclusion is further supported by the observations that (a) neither production of ethylene nor senescence of detached rice leaves was affected by DFMO and DFMA, and (b) treatments with DCH and MGBG, which resulted in an increase in the production of ethylene, promoted senescence of detached rice leaves. Since DCH and MGBG reduced levels of Spm and increased those of Put in rice leaves, the stimulatory effect of DCH and MGBG on the production of ethylene is most likely mediated by the redirection of a portion of the pool of SAM into the ethylene pathway. Characterization of the $\mathrm{DCH}$ - and MGBG-induced production of ethylene will be published elsewhere.
This work was supported financially by the National Science Council of the Republic of China (NSC 79-0409-B002-04). We thank Dr. Peter McCann and the Merrell Dow Research Institute, incinnati, Ohio, for the gift samples of DFMO and DFMA.

\section{References}

Altman, A. (1982) Retardation of radish leaf senescence by polyamines. Physiol. Plant. 54: 189-193.

Batchelor, K. W., Smith, R. A. and Watson, N. S. (1986) Dicyclohexylamine is not an inhibitor of spermidine synthase. Biochem. J. 233: 307-308.

Biondi, S., Torrigiani, P., Sansovini, A. and Bagni, N. (1988) Inhibition of polyamine biosynthesis by dicyclohexylamine in cultured cotyledons of Pinus radiata. Physiol. Plant. 72: 471476.

Birecka, H., Birecki, M. and Ireton, K. (1990) Endogenous polyamine levels and dark-induced leaf senescence. Plant Physiol. 93: S-757.

Birecka, H., DiNolfo, T. E., Martin, W. B. and Frohlich, M. W. (1984) Polyamines and leaf senescence in pyrolizidine alkaloidbearing Heliotropium plants. Phytochemistry 23: 991-997.

Carbonell, J. and Navarro, J. C. (1989) Correlation of spermine levels with ovary senescence and with fruit set and development in Pisum sativum L. Planta 178: 482-487.

Chen, C. T., Chou, I. T. and Kao, C. H. (1990) Senescence of rice leaves $\mathrm{XX}$. Changes of proton secretion during senescence. Plant Sci. 66: 29-34.

Cheng, S. H. and Kao, C. H. (1983) Localized effect of polyamines on chlorophyll loss. Plant Cell Physiol. 24: 1463-1467.

Evans, P. T. and Malmberg, R. L. (1989) Do polyamines have roles in plant development? Annu. Rev. Plant Physiol. Plant Mol. Biol. 40: 235-269.

Feirer, R. P., Wann, S. R. and Einspahr, D. W. (1985) The effects of spermidine synthesis inhibitors on in-vitro plant development. Plant Growth Regul. 3: 319-327.

Flores, H. E. and Galston, A. W. (1982) Analysis of polyamines in higher plants by high performance liquid chromatography. Plant Physiol. 69: 701-706.

Galston, A. W., Altman, A. and Kaur-Sawhney, R. (1978) Polyamines, ribonuclease and the improvement of oat leaf protoplasts. Plant Sci. Lett. 11: 69-79.

Hemantaranjan, A. and Garg, O. K. (1984) Effects of cytokinins and several inorganic cations on the polyamine content of (Hordeum vulgare L.) endosperms. Indian J. Exp. Biol. 22: 379-381.

Hibasami, H., Tanaka, M., Nagai, J. and Ikeda, T. (1980) Dicyclohexylamine, a potent inhibitor of spermidine synthetase in mammalian cells. FEBS Lett. 116: 99-101.

Hirawasa, E. and Suzuki, Y. (1983) Biosynthesis of spermidine in maize seedlings. Phytochemistry 22: 103-106.

Kao, C. H. (1978) Senescence of rice leaves II. Antisenescent action of cytokinins. Proc. Natl. Sci. Counc. ROC 2: 391-398.

Kao, C. H. and Yang, S. F. (1983) Role of ethylene in the senes- 
cence of detached rice leaves. Plant Physiol. 73: 881-885.

Kaur-Sawhney, R. and Galston, A. W. (1979) Interaction of polyamines and light on biochemical processes involved in leaf senescence. Plant Cell Environ. 2: 189-196.

Kaur-Sawhney, R., Shih, L.M., Flores, H. E. and Galston, A. W. (1981) Relation of polyamine synthesis and titer to ageing and senescence in oat leaves. Plant Physiol. 69: 405-410.

Lin, P. P. C. (1984) Polyamine metabolism and its relation to response of the aleurone layers of barley seeds to gibberellic acid. Plant Physiol. 74: 975-983.

Lowry, O. H., Rosebrough, N. J., Farr, A. L. and Randall, R. J. (1951) Protein measurement with the Folin-phenol reagent. $J$. Biol. Chem. 193: 265-275.

Mitchell, J. L. A., Wahan, D. W., McCann, P. P. and Qasba, P. (1985) Dicyclohexylamine effect on HTC cell polyamine content and ornithine decarboxylase activity. Biochim. Biophys. Acta 840: 309-315.

Miyazaki, J. and Yang, S. F. (1987) The methionine salvage pathway in relation to polyamine biosynthesis. Physiol. Plant. 69: 366-370.

Roberts, D. R., Walker, M. A., Thompson, J. E. and Dumbroff, E. B. (1984) The effects of inhibitors of polyamines and ethylene biosynthesis on senescence, ethylene production and polyamine levels in cut carnation flowers. Plant Cell Physiol. 25: 315-322.

Shibaoka, H. and Thimann, K. V. (1970) Antagonism between kinetin and amino acids: experiments on the mode of action of cytokinins. Plant Physiol. 46: 212-220.

Srivastava, S. K., Raj, D. S. and Naik, B. I. (1981) Polyamine metabolism during ageing $\&$ senescence of pea leaves. Indian J. Exp. Biol. 19: 437-440.

Suresh, M. R. and Adiga, P. R. (1978) Absence of parallelism between polyamine and nucleic acid contents during induced growth of cucumber cotyledongs. Biochem. J. 172: 185-188.

Suresh, M. R., Ramakrishna, S. and Adiga, P. R. (1978) Regulation of arginine decarboxylase and putrescine levels in Cucumis sativus cotyledons. Phytochemistry 17: 57-63.

Torrigiani, P., Serafini-Fracassini, D. and Bagni, N. (1987) Polyamine biosynthesis and effect of dicyclohexylamine during the cell cycle of Helianthus tuberosus tuber. Plant Physiol. 84: 148-152.

Walker, M. A., Roberts, D. R. and Dumbroff, E. B. (1988) Effects of cytokinin and light on polyamines during the greening response of cucumber cotyledons. Plant Cell Physiol. 29: 201205.

Walker, M. A., Roberts, D. R., Shih, C. Y. and Dumbroff, E. B. (1985) A requirement for polyamines during the cell-division phase of radicle emergence in seeds of Acer saccharum. Plant Cell Physiol. 26: 967-971.

Williams-Ashman, H. and Schenone, A. (1972) Methylglyoxal bis-(guanylhydrazone) as a potent inhibitor of S-adenosylmethionine decarboxylases. Biochem. Biophys. Res. Commun. 46: 288-295.

Wintermans, J. F. G. M. and De Mots, A. (1965) Spectrophotometric characteristics of chlorophylls $\mathrm{a}$ and $\mathrm{b}$ and their pheophytins in ethanol. Biochim. Biophys. Acta 109: 448453.

(Received March 30, 1991; Accepted June 27, 1991) 
